\title{
Extra cardiac tumor misdiagnosed as a left atrial myxoma
}

\author{
Kwangook Choi ${ }^{1}$, Dongho Jung ${ }^{1}$, Seong Wook Hong ${ }^{1}$, Younghoon Jeon ${ }^{2}$, and Si Oh Kim ${ }^{1}$ \\ Department of Anesthesiology and Pain Medicine, ${ }^{1}$ Kyungpook National University School of Medicine, ${ }^{2}$ Kyungpook National \\ University School of Dentistry, Daegu, Korea
}

Cardiac tumors, which are characterized by very a low prevalence of approximately $0.02 \%$ based on pooled data, can cause considerable morbidity and mortality due to cardiac conduction abnormalities, embolization of the tumor fragment or associated thrombus, or intra-cardiac obstructions [1]. Primary cardiac tumors are usually benign in adults, with only $20-25 \%$ cases being reported as malignant, and myxoma is the most common cardiac neoplasm, accounting for nearly $50 \%$ of cases of cardiac tumors. Myxomas most commonly originate in the left atrium; however, sarcomas may also present as a left atrial (LA) mass, and may be mistaken for a myxoma, preoperatively. For myxomas, surgical resection is adopted as a curative treatment, and transesophageal echocardiography (TEE) is currently the most powerful cardiovascular monitoring technique available in the perioperative medicine setting; it plays a vital role in the assessment of cardiac surgical plans and in anesthetic management [2].
Herein, we report the case of a patient with a malignant extra cardiac tumor that was misdiagnosed as an LA myxoma; in this case, intra-operative TEE played a pivotal role in facilitating the completion of the resection during cardiac surgery.

A $3 \mathrm{~cm}$ mass or thrombus-like lesion within the LA cavity was detected incidentally, upon chest computed tomography (CT) in a 51-year-old woman (Fig. 1A). A subsequent transthoracic echocardiographic examination (TTE) also revealed the presence of an echogenic mass, with characteristic features of a myxoma in the left atrial appendage (LAA), and concomitant mild mitral valve regurgitation, but normal ejection fraction (59\%). An operation, involving median sternotomy under the general anesthesia, including minimally invasive techniques and a cardiopulmonary bypass (CPB), was performed with the aim of resecting the presumed LA myxoma. The anesthetic management proceeded uneventfully, with $0.5 \%$ sevoflurane, and
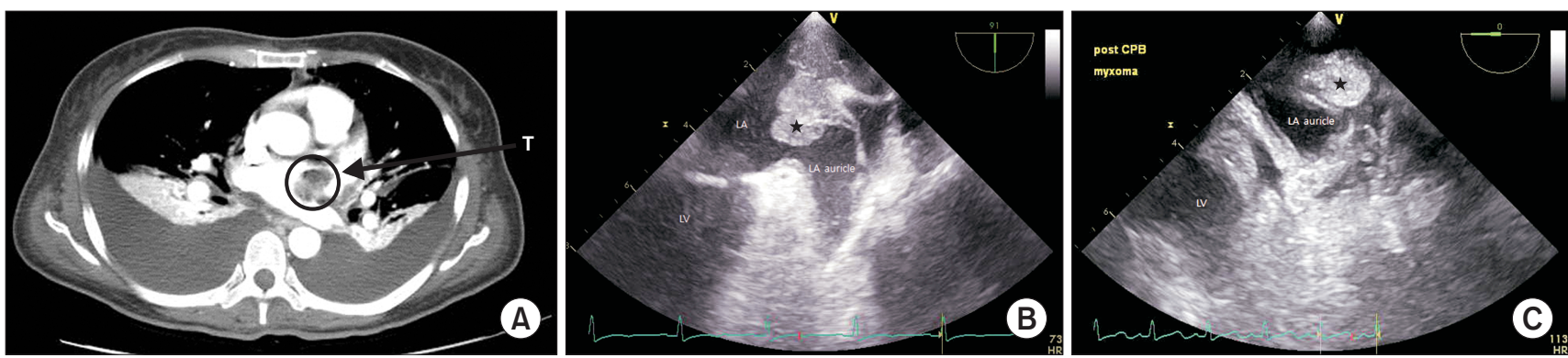

Fig. 1. Computerized tomography of the chest revealed a cardiac mass (T) at the anterior portion of the left atrium (A). Transesophageal echocardiographic, midesophageal two-chamber view prior to the initiation of cardiopulmonary bypass. The mass $(\star)$ was observed in the left atrial appendage (B). Transesophageal echocardiographic, midesophageal two-chamber view after cardiopulmonary bypass. The mass ( $\star$ ) was still observed in the left atrial appendage (C). LA: Left atrium, LV: Left ventricle.

Corresponding author: Si Oh Kim, M.D., Ph.D., Department of Anesthesiology and Pain Medicine, Kyungpook National University School of Medicine, 130, Dongdeok-ro, Jung-gu, Daegu 700-721, Korea. Tel: 82-53-420-5863, Fax: 82-53-426-2760, E-mail: sokim@knu.ac.kr

(c) This is an open-access article distributed under the terms of the Creative Commons Attribution Non-Commercial License (http:// creativecommons.org/licenses/by-nc/3.0/), which permits unrestricted non-commercial use, distribution, and reproduction in any medium, provided the original work is properly cited. 
continuous infusion of fentanyl with rocuronium was administered. Prior to CPB initiation, the TEE examinations detected a mobile mass between the LAA and left pulmonary vein, which confirmed the echocardiographic findings of the preoperative TTE examinations (Fig. 1B). The surgery aimed to remove the mass, with concomitant mitral valvuloplasty; the excised mass $(2 \times 2 \mathrm{~cm}$ in size $)$ was gelatinous and mobile, and was attached to the wall between the LAA and left upper pulmonary vein. However, after rewarming and repairing the left atriotomy, the post-CPB TEE examinations revealed that the tumor was still present within the LA cavity (Fig. 1C). After discussion with the surgeons, the mass was inspected once more via a left atriotomy; however, visual inspection did not indicate any tumor within the cavity. Conversely, the TEE revealed that the mass was still located in the LA. It was concluded that the mass was of an extra-cardiac origin; subsequently, re-weaning from the $\mathrm{CPB}$ was performed uneventfully. The patient awoke 4 hours after arriving at the intensive care unit, with no neurological deficits; she was discharged uneventfully on POD 15. A postoperative chest CT only revealed mural thrombi at the anterior aspect of the LA; however, the LAA was not observed. Conversely, the postoperative TTE did not reveal any mass in the LA cavity, and an ejection fraction of $46 \%$ was recorded. Histopathological analysis indicated an undifferentiated epithelioid sarcoma, which appeared to be a malignant fibrous histiocytoma (MFH) upon microscopy. The metastatic workup was negative, and the patient subsequently underwent chemotherapy.

Preoperatively, most cardiac masses are considered to be benign, and thus, a diagnosis of sarcoma is often made during or after surgery, based on the tumor characteristics or pathology. Furthermore, almost all malignant tumors occur preferentially on the right side of the heart; however, we report a case of a primary cardiac sarcoma in the left atrium, mixed with cardiac myxoma. Some echocardiographic characteristics may help dif- ferentiate an MFH from an atrial myxoma; of these, one of the most common features is the site of its origin. Atrial myxomas are usually attached to the fossa ovalis, but may arise from the mitral or tricuspid valves, or even the right atrium [3]. Conversely, MFHs commonly originate near the pulmonary veins or the LAA. Moreover, the attachment of an MFH to the endocardium is typically broad-based, thus differentiating them from typical atrial myxomas, which are commonly attached via a stalk or a pedicle [4]. In the present case, upon TEE examination prior to $\mathrm{CPB}$, the mass was observed in the LAA, extending from the left upper pulmonary vein. A more observant diagnosis, based on the TEE findings at this point, might have reduced the unnecessary expenses. In this case, the mass in the LA cavity that was identified by TEE examination after CPB was considered to be a primary cardiac tumor originating from the epicardium. Therefore, the mass that was removed was only a small portion of the tumor that invaded the LAA. After rewarming, another search was conducted for the remnant mass through a left atriotomy, however, no tumor was found within the cavity. This might also be explained by the extra-cardiac origin nature of the mass.

Irrespective of the cause or location of the tumor, a comprehensive TEE examination during cardiac surgery remains the best method for detecting intra-cardiac tumors, and can provide the surgeon with the information needed to finalize the surgical plan and to evaluate the successful excision of the cardiac mass and achieve minimal damage to adjacent structures during excision [5]. This case confirms that tumors of extra-cardiac origin may also occur, and hence, this possibility should be considered when the location or shape of the mass lesion is unusual on preCPB TEE examinations, or when remnant mass-like lesions are noted on post-CPB TEE examinations during LA myxoma surgeries.

\section{References}

1. Reynen K. Cardiac myxomas. N Engl J Med 1995; 333: 1610-7.

2. Blackmon SH, Patel A, Reardon MJ. Management of primary cardiac sarcomas. Expert Rev Cardiovasc Ther 2008; 6: 1217-22.

3. Harris GJ, Tio FO, Grover FL. Primary left atrial myxosarcoma. Ann Thorac Surg 1993; 56: 564-6.

4. Burke AP, Cowan D, Virmani R. Primary sarcomas of the heart. Cancer 1992; 69: 387-95.

5. Kośmider A, Jaszewski R, Marcinkiewicz A, Bartczak K, Knopik J, Ostrowski S. 23-year experience on diagnosis and surgical treatment of benign and malignant cardiac tumors. Arch Med Sci 2013; 9: 826-30. 